\title{
Illumination Invariant Face Recognition
}

\author{
Hardeep Kaur \\ Department of Computer Science \\ Punjabi University \\ Patiala, India
}

\author{
Amandeep Kaur \\ Assistant Professor \\ Department of Computer Science \\ Punjabi University, Patiala, India
}

\begin{abstract}
Face Recognition is a term that includes several subproblems. Though face recognition have been a grown up research area, however, there still remain many problems that must be overcome to develop a robust face recognition system that works well under various circumstances such as illumination, pose and expressions variations. Such variations have proven to be one of the biggest problems of face recognition systems. In the proposed thesis work the problem of illumination is discussed. A method based on the combination of Retinex and LOG-DCT technique is applied to suppress the illumination and for better face recognition results. After illumination normalization LBP is used for extracting the features of normalized images, which are further used for face recognition. In the proposed method the experiments on Extended Yale B database show that by using the proposed method better recognition performance and results can be obtained.
\end{abstract}

Keywords: Face Recognition, Retinex, LOG-DCT, normalization, LBP.

\section{INTRODUCTION AND RELEVANT PREVIOUS WORK}

There are enormous applications where a person's identity is important. For the same, there are many different identification technologies available, many of which have been in commercial use for years. Some of those methods are fingerprints, retina recognition, and iris recognition and face recognition etc.

Despite the success of many face recognition systems many issues remain to be addressed. Among those issues the non linear illumination problem is prominent for most systems. In case of non linear illumination problem the same face appears differently due to change in lighting. More specifically the changes induced by illumination could be larger than the differences between the individuals, causing systems based on comparing images to misclassify the identity of the input image. In [4] an extensive and up-to-date survey of the existing techniques to address illumination problem is presented. Broadly illumination supression tecniques are divided into two parts firstly illumination invariant feature extraction tecniques and secondly preprocessing and normalization tecniques First category includes logarithmic transformation, edge map, and image gradient [1] for face recognition. These algorithms are easy to implement but the improvement in terms of the recognition rate is very limited. Recently, in [8] a novel technique called Gradient faces is applied by extracting the illumination insensitive measure from the gradient domain. The extraction of illumination invariant features from frequency domain was implemented using wavelet transform in Wavelet face [11], using discrete cosine transform (DCT) [10], and using discrete Fourier transform (DFT) [12]. Many subspace-based methods have also been proposed, such as self quotient image (SQI) [7]. These methods are effective to suppress the illumination but large numbers of training samples are always required in these methods.

In many existing techniques, only the small-scale features are extracted for face recognition in order to avoid the illumination variation problem. Such methods mainly include Land's Retinex model [13] and its variants. Based on the Retinex theory, a face image is decomposed into its smoothed version and its illumination invariant features. To obtain such decomposition, the total variation model, Gauss filtering, weighted Gauss filtering, and wavelet transform are employed in logarithmic total variation (LTV) model [14], self quotient image (SQI) [15], respectively. Among the methods of extracting the illumination insensitive/invariant features, the Retinex theory-based methods [13-21] always perform better than the others. However, the large-scale features of a face image, which may also contain useful information for recognition, are always discarded in these methods. A novel method of normalizing both the Small-and Large-scale (S\&L) features of a face image is proposed in [17].

Second category includes methods of pre-processing and normalization techniques in which face images are preprocessed using some image processing techniques to normalize the images to appear stable under different lighting conditions. Early algorithms are used to make a simple grayscale adjustment on the face image, e.g., Gamma correction, histogram equalization (HE), and histogram match (HM) [18]. Typical algorithms include morphing face, linear lighting model-based illumination compensation, shadow compensation, and illumination compensation by truncating low frequency coefficients of DCT in the logarithm domain [19]. But a strict alignment upon a fixed shape model is always needed in these algorithms. However, strict alignment is also challenging under varying illuminations.

In the proposed research work, small- and large- scale(S\&L) [17] based technique with the combination of Retinex and LOG-DCT is proposed for illumination compensation and LBP is used for extracting the features of normalized image for further recognition process.

\section{METHODOLOGY}

In this paper normalized images by using the combination of retinex and LOG-DCT method used with the current LBPbased face recognition system, so it makes full use of advantages of the illumination compensation offered by the large and small scale features [17], as well as the powerful discrimination ability provided by the LBP representation [20]. Extended Yale B database is selected for the experiments. 10 individual's images are selected captured 
under 50 lighting conditions from different viewpoints. All the images are manually cropped to the size of $170 \times 170$. All the images are divided into 5 subsets according to the lighting angle. Following table 1 shows the subsets of $10 \times 50(500)$ images:

Table 1. Table captions should be placed above the table

\begin{tabular}{|l|c|c|c|c|c|}
\hline Subset & 1 & 2 & 3 & 4 & 5 \\
\hline $\begin{array}{l}\text { Lighting } \\
\text { angle }\end{array}$ & $0 \sim 12$ & $13 \sim 25$ & $26 \sim 50$ & $51 \sim 77$ & $>77$ \\
\hline
\end{tabular}

Sample images of an individual from Extended Yale B Database divided into five subsets are shown in following figure:

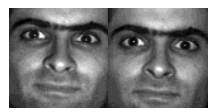

Subset 1

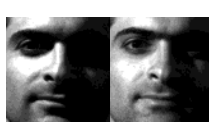

subset 4

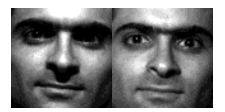

Subset 2

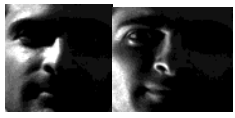

Subset 5

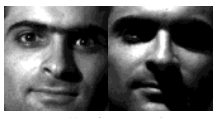

Subset 3
Fig 1: Sample images of an individual according to lighting angle divided into five subsets.

The proposed method consists of following steps:

1. Decomposing an image into large- and small- scale features.

2. Applying thresh holding filter on small scale features

3. Applying normalization technique( LOG-DCT) on large scale features

4. Feature Extraction by using LBP

5. Face Matching

\subsection{Decomposition}

Retinex method [13-21] is used for image decomposition. The input face image is decomposed as $\mathrm{R}$ and $\mathrm{L}$ respectively Reflectance and Illumination by applying retinex method.

\section{STEP 1}

Retinex algorithm decomposes a given image $S$ into two images: the reflectance image $R$, and the illumination image $L$, such that each point in the image domain $S(x, y)$ can be expressed as Equation:

STEP 2

$$
S(x, y)=R(x, y) . L(x, y)
$$

In second step the image is taken to the logarithmic domain, which gives:

where

$$
s=l+r
$$

$$
s=\log S, l=\log L \text { and } r=\log R
$$

The reflectance is restricted to the range $R \in[0,1]$, and $L \geq S$, which implies $1 \geq \mathrm{s}$. Thus the retinex algorithm is used to reduce the image into reflectance and illumination image. The two iterations of the algorithms are applied on the original and inverted image to give bright and dark retinex which reveal more information from the original image. Following figure shows the input image and the resulted output images after applying retinex method.

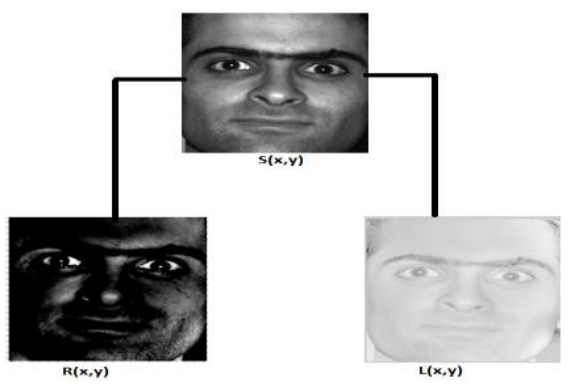

Fig 2: S(x,y) Original Image, $R(x, y)$ Reflectance Image, $\mathbf{L}(\mathbf{x}, \mathbf{y})$ Illumination Image

\subsection{Smoothing on Small Scale Features}

Since the estimation of reflectance is an ill-posed problem, the retinex decomposition may also bring in some undesirable results we perform a threshold-average filtering on $\mathrm{R}$ to enhance the visual results. Figure 3 shows the small scale features $\mathrm{R}(\mathrm{x}, \mathrm{y})$ and smoothed version of $\mathrm{R}, \mathrm{Rs}(\mathrm{x}, \mathrm{y})$.
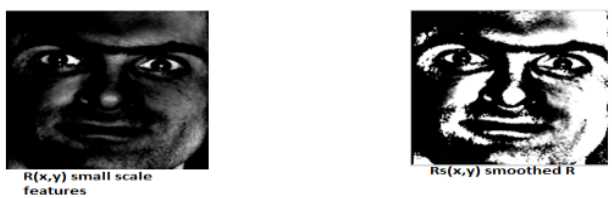

Fig 3: $R(x, y)$ small scale features, $\operatorname{Rs}(x, y)$ smoothed $R$

\subsection{Illumination Normalization on Large Scale Features}

LOG-DCT was developed based on the theory that illumination variations mainly lie in the low-frequency band. It has been suggested in [19] that the appropriate number of DCT coefficients in the logarithm domain can be used to approximate the illumination variations, and that these DCT coefficients can then be truncated to reduce the effect of illumination variation for face recognition. Here we use LOGDCT algorithm for pre-processing the large-scale features.
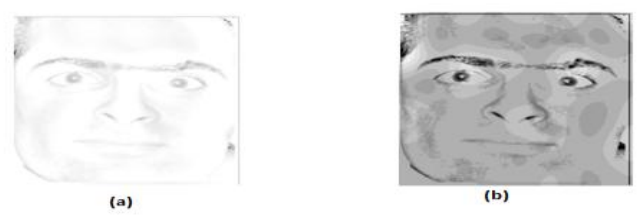

Fig 4: (a) $L(x, y)$ Large scale features (b) $L$ normalized by LOG-DCT (i.e. RI)

General steps of applying LOG-DCT are explained below:

\section{STEP I}

A face image $\mathrm{s}$ is assumed to be proportional to the product of the reflectance $r(x, y)$ and the illumination $l(x, y)$, i.e.

$$
s(x, y)=r(x, y) . l(x, y)
$$

Since the reflectance is a stable characteristic of facial features, the goal is to recover the reflectance of faces under varying illumination conditions. Taking logarithm transform on (1) we get:

$$
\log s(x, y)=\log r(x, y)+\log l(x, y)
$$


It follows from (4) that in the logarithm domain, if the incident illumination $l(x, y)$ and the desired uniform illumination $e$ are given ( $e$ is identical for every pixel of an image), we have:

$$
\begin{gathered}
\log f^{\prime}\left(x_{v} y\right)=\log r(x, y)+\log e^{\prime} \\
\log r\left(x_{v} y\right)+\log e\left(x_{v} y\right)-\in\left(x_{v} y\right) \\
\log f\left(x_{v} y\right)-\in\left(x_{v} y\right)
\end{gathered}
$$

where

$$
E\left(x_{v} y\right)=\log e\left(x_{i} y\right)-\log e^{\prime}
$$

and $f^{*}(x, y)$ is the pixel value under desired uniform illumination. From (3), we can conclude that the normalized face image can be obtained from the original image by using an additive term $\boldsymbol{E}(\boldsymbol{x}, \boldsymbol{y})$ called compensation term which is the difference between the normalized illumination and the estimated original illumination in the logarithm domain.

\section{STEP II}

\section{Applying DCT:}

There are four established types of discrete cosine transforms (DCTs), i.e., DCT-I, DCT-II, DCT-III and DCT-IV. The DCTII is more widely applied in signal coding, hence, it is often simply referred to as "the DCT". The 2D $M \times N$ DCT is defined as follows:

$$
\begin{aligned}
& \mathrm{C}\left(u_{v}, v\right)=\alpha(u) \alpha(v) \sum_{x=0}^{M-1} \sum_{y=0}^{N-1} f\left(x_{v} y\right) \\
& \quad \mathrm{x} \cos \left[\frac{\pi(2 x+1) u}{2 M}\right] \cos \left[\frac{\pi(2 y+1) v}{2 N}\right]
\end{aligned}
$$

And inverse transform is defined as:

$$
\begin{aligned}
& f(x, y)=\sum_{u=0}^{M-1} \sum_{v=0}^{N-1} \alpha(u) \alpha(v) C(u, v) \\
& \quad \times \cos \left[\frac{\pi(2 x+1) u}{2 M}\right] \cos \left[\frac{\pi(2 y+1) v}{2 N}\right]
\end{aligned}
$$

Where:

$$
\begin{gathered}
\alpha(u)=\frac{1}{\sqrt{M}^{\prime}} \text { if } u=0 \\
\alpha(u)=\sqrt{\frac{2}{M}}, \text { if } u=1,2, M-1 \\
\alpha(v)=\frac{1}{\sqrt{N}}, \text { if } v=0 \\
\alpha(v)=\sqrt{\frac{2}{N}}, \text { if } v=1,2 \ldots N-1
\end{gathered}
$$

\section{STEP III}

\section{Illumination compensation:}

Given a face image, illumination variations can be well compensated by adding or subtracting the compensation term $\in(x, y)$ of (6) in the logarithm domain. If $l$ lowfrequency DCT coefficients are set to zero (in the same manner described in [19], we have

$$
\begin{aligned}
F^{\prime}\left(x_{v} y\right) & =\sum_{\tilde{L}=0}^{M-1} \sum_{\mathbb{V}=0}^{N-1} E\left(u_{v} v\right)-\sum_{i=1}^{n} E\left(u_{v} v\right) \\
& =F\left(x_{v} y\right)-\sum_{i=1}^{n} E\left(u_{i_{s}} v_{i}\right)
\end{aligned}
$$

$E\left(u_{v} v\right)=\alpha(u) \alpha(v) C\left(u_{v} v\right) \cos \left[\frac{\pi(2 x+1) u}{2 M}\right] \cos \left[\frac{x(2 y+1) v}{2 N}\right](10)$

The term $\sum l_{i=1} E\left(u_{i}, v_{i}\right)$ is the illumination compensation term. $F^{\prime}\left(x_{v} y\right)$ In (9) is the desired normalized face in the logarithm domain.

\subsection{Reconstruction}

The normalized face image is finally reconstructed by combining the normalized large-scale features $\mathrm{Rl}$ and smoothed small scale features Rs.

$$
I_{\text {norm }}(x, y)=R s(x, y) R l(x, y)
$$

Reconstructed image from $\mathrm{Rs}$ and $\mathrm{Rl}$ is shown in the following Figure 5.

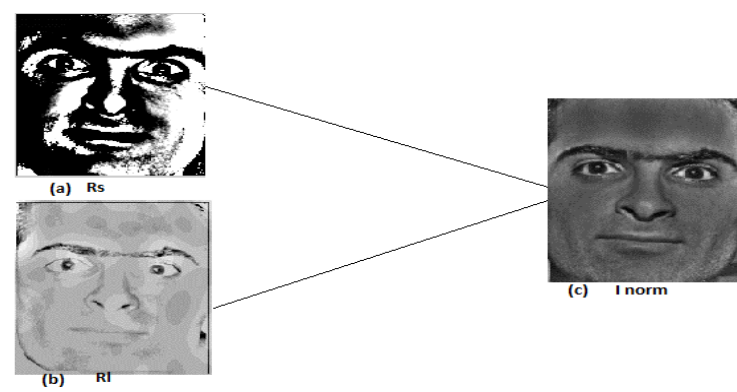

Fig 5: (a) shows the smoothed small scale features Rs (b) shows the normalized large scale features $\mathrm{RI}(\mathrm{c})$ is the reconstructed image $\mathbf{I}_{\text {norm. }}$.

\subsection{LBP Feature Extraction}

$\mathrm{I}_{\text {norm }}$ is the normalized image which is further used feature extraction by using LBP. In the proposed research work Simple LBP method and Euclidean distance is used for feature extraction and Image recognition. The equation of LBP is as shown below:

$$
L B P=\sum_{p=0}^{7} 2^{p} \times S\left(g_{p}-g_{c}\right)
$$

By applying (12) on normalized images LBP features will be extracted via histogram. These histogram values will further use for the image matching.

\subsection{Feature Matching}

For feature matching here we use very basis technique Euclidean Distance. The general formula for Euclidean distance is:

$$
\begin{aligned}
& d(p, q)=d(q, p)= \\
& \sqrt{\left(q_{1}-p_{1}\right)^{2}+\left(q_{2}-p_{2}\right)^{2}+\cdots+\left(q_{n}-p_{n}\right)^{2}}= \\
& \sqrt{\sum_{i=1}^{n}\left(q_{i}-p_{i}\right)^{2}}
\end{aligned}
$$

The resulted histogram values extracted during feature extraction phase of LBP are used to compute minimum distance. The resulted data is stored in the different array, which will give the minimum value at the end for a single test image. And hence the minimum value will give the best possible match. Similarly, the other test images can also be

Where 
tested with train images in the matching process. The minimum distance will be the best matching image.

\section{RESULTS}

The proposed method is evaluated on Extended Yale B face database. The database is divided into 5 subsets according to the lighting angle. Subset 1 contains the test images which are tested on subset 2, 3, 4 and 5 respectively. The proposed method gives good results as compared to related state- ofthe- art methods. The experiments show that proposed method gives good results as compared to simple LOG-DCT and LBP technique. The Proposed method is based on the combination of Retinex and LOG (DCT) method and LBP. The implementation results of the proposed method are shown in the following table 2 :

Table 2. Recognition Rate (\%) and Comparison with other Methods

\begin{tabular}{|l|c|c|c|c|}
\hline \multirow{2}{*}{ Method } & \multicolumn{4}{|c|}{ Recognition Rate (\%) } \\
\cline { 2 - 5 } & subset 2 & subset 3 & subset 4 & subset 5 \\
\hline $\begin{array}{l}\text { Single scale } \\
\text { Retinex and } \\
\text { LBP }\end{array}$ & $76 \%$ & $64 \%$ & $34 \%$ & $20 \%$ \\
\hline $\begin{array}{l}\text { Multi Scale } \\
\text { Retinex and } \\
\text { LBP }\end{array}$ & $79 \%$ & $53 \%$ & $40 \%$ & $10 \%$ \\
\hline $\begin{array}{l}\text { LOG(DCT)an } \\
\text { d LBP }\end{array}$ & $80 \%$ & $75 \%$ & $72 \%$ & $69 \%$ \\
\hline $\begin{array}{l}\text { R\&LOG(DC } \\
\text { T) LBP }\end{array}$ & $92 \%$ & $90 \%$ & $80 \%$ & $60 \%$ \\
\hline
\end{tabular}

Experiments results on Extended Yale B Data Base shows that the total recognition rates of Single Scale Retinex and LBP, Multi scale Retinex and LBP, LOG-(DCT) and LBP method and Retinex based LOG(DCT) and LBP method are respectively $48 \%, 45 \%, 74 \%$ and $82 \%$. Thus the proposed method gives good results as compared to related state- ofthe- art methods. Other advantage of this method is its simplicity so it is very suitable of real time manipulation.

\section{CONCLUSION AND FUTURE WORK}

In the proposed research work a technique based on the combination of Retinex and LOG(DCT) method is introduced for illumination suppression, images normalized with proposed method when used with LBP feature extraction method then it gives good recognition results as compared to other retinex methods but results are slightly down as compared to Small scale and large scale technique proposed in[17]. In the proposed research work the proposed method is applied on Extended Yale B database with simple LBP feature extraction technique. The future work include the use of proposed method on more face databases and with other advanced LBP techniques for further improvement of results.

\section{REFERENCES}

[1] Adnin Y., Moses Y, and Ullman S. 1997. Face recognition: The problem of compensating for changes in illumination direction. IEEE Transactions on Pattern Analysis and Machine Intelligence, Vol. 19, No. 7.
[2] Kouzani A. Z., He F., Sammut K., and Bouzerdoum A. 1998. IIIumination Invariant Face Recognition.IEEE Conference, pp. 4240 - 4245 vol.5.

[3] Jiang L., Fang B., Zhang T., Tang. Y. Y., and Li D. H. 2009. Face Recognition Under Varying Illumination Using Adaptive Filtering. International Conference on Wavelet Analysis and Pattern Recognition, (ICWAPR), pp. 38-42.

[4] Zou X., Kittler J. and Messer K. 2007. Illumination Invariant Face Recognition: A Survey. First IEEE International Conference on Biometrics Theory Applications and Systems.

[5] Savvides M. and Kumar V. 2003. Illumination normalization using logarithm transforms for face authentication, in Proc. IAPR AVBPA, pp.549-556.

[6] Xie X.D. and Lam K. M. 2006. An Efficient Illumination Normalization Method for Face Recognition. Pattern Recognition Letters, 27(6): 609-617.

[7] Wang H., Li S. Z., and Wan Y. 2004. Face Recognition under Varying Lighting Conditions Using Self Quotient Image. IEEE International Conference on Automatic Face and Gesture Recognition (FGR'04) 0-7695-2122$3 / 04$.

[8] Zhang T., Tang Y., Fang Y., Shang Z., and Liu X. 2009. Face Recognition under Varying Illumination Using Gradient faces. IEEE Transactions on Image Processing, Vol. 18, No. 11, pp. 2599-2606.

[9] Shan S., Gao W., Cao B., and Zhao D. 2003. Illumination normalization for robust face recognition against varying lighting conditions. In Proc. IEEE Workshop on AMFG, pp. 157-164.

[10] Hafed Z. and Levine M. 2001. Face recognition using the discrete cosine transform. Int. J. Comput. Vis, vol. 43, no. 3, pp. $167-188$.

[11] Garcia C., Zikos G. and Tziritas G. 1998A wavelet-based framework for face recognition. In Proc. Eur. Conf. Comput. Vis., Freiburg, pp. 84-92.

[12] Lai J., Yuen P. C. and Fengc G. 2001. Face recognition using holistic Fourier invariant features. Pattern Recognit., vol. 34, pp. 95-109.

[13] Land E. H. and McCann J. J. 1971. Lightness and retinex theory. J. Opt. Soc. Amer., vol. 61, no. 1, pp. 1-11.

[14] Chen T., Yin W., Zhou X. S., Comaniciu D. and Huang T. S. 2006. Total variation models for variable lighting face recognition. IEEE Trans. Pattern Anal. Mach. Intell, vol. 28, no. 9, pp. 1519-1524.

[15] Wang H., Li S. Z., and Wang Y. 2004. Face recognition under varying lighting conditions using self quotient image. In Proc. Conf. Autom. Face Gesture Recognition., Seoul, pp. 819-824.

[16] Zhang T, Gang B., Yuan Y., Tang Y. Y., Shang Z., Li D., and Lang F. 2009. Multiscale facial structure representation for face recognition under varying illumination. Pattern Recognition. vol. 42, no. 2, pp. 251-258.

[17] Xie X., Zheng W. S., Lai J., Yuen P. C., and Suen C. Y. 2011. Normalization of Face Illumination Based on 
Large-and Small-Scale Features. IEEE Transaction on Image Processing, Vol. 20, No. 7.

[18] Pizer S. M. and Amburn E. P. 1987. Adaptive histogram equalization and its variations. Comput. Vis. Graph., Image Process, vol. 39, no. 3, pp. 355-368.

[19] Chen W., Meng J. E. and Wu s. 2006. Illumination Compensation and Normalization for Robust Face Recognition Using Discrete Cosine Transform in Logarithm Domain. IEEE Transaction on Systems, Man, And Cybernetics - Part B: Cybernetics, VOL. 36, NO. 2.
[20] Hong P., Si-Yu X., Li-Zuo J., Liang-Zheng X. 2011. Illumination Invariant Face Recognition Based on Improved Local Binary Pattern. IEEE, 30th Chinese Control Conference (CCC), pp. 3268-3272.

[21] Jobson D. J, Rahman Z., and Woodell G. A. 1997. Properties and Performance of a Center/Surround Retinex. IEEE Transactions on Image Processing, vol. 6, no. 3, pp. 451-462.

[22] http://www.face-rec.org/ 